\title{
Relación entre atención compartida madre-hijo, sensibilidad materna y expresión emocional gestual infantil
}

\author{
Elizabeth Muñoz Londoño* y Chamarrita Farkas. \\ Pontificia Universidad Católica de Chile
}

Recibido, junio 1/2017

Concepto de evaluación, julio 24/2017

Aceptado, septiembre 26/2017
Referencia: Muñoz Londoño, E. \& Farkas, C. (2018). Relación entre atención compartida madre-hijo(a), sensibilidad materna y expresión emocional gestual infantil. Acta colombiana de Psicología, 21(2), 131-143. doi: http://www.dx.doi.org/10.14718/ACP.2018.21.2.7

Resumen

\begin{abstract}
La atención compartida se refiere a episodios en los que el niño y su cuidador están enfocados intencionalmente en algún objeto o actividad, presentando intercambios físicos y emocionales. En este estudio se describió la atención compartida considerando las dimensiones de nivel de compromiso y tono emocional, y se analizó su relación con la sensibilidad materna y la intensidad de la expresión emocional gestual de niños de un año de edad. La muestra estuvo compuesta por 12 díadas madre-hijo, donde se consideró como criterios de inclusión que los niños tuvieran entre 12 y 14 meses de edad, que fueran hijos únicos, que vivieran con ambos padres, y que asistieran a sala cuna. Los instrumentos utilizados fueron la Evaluación de Atención Compartida, la Evaluación de la Expresión Emocional Gestual del Niño y la Escala de Sensibilidad del Adulto. Los resultados evidencian episodios de atención compartida en díadas madre-hijo al año de edad, además de una relación significativa entre atención compartida con el nivel de compromiso y la sensibilidad materna.

Palabras clave: atención compartida, expresión emocional gestual, interacción madre-niño, sensibilidad materna.
\end{abstract}

\section{Relationship between mother-child shared attention, maternal sensitivity and infant emotional gestural expression}

\begin{abstract}
Shared attention refers to episodes through which a child and his or her caretaker are intentionally focused on some object or activity while engaging in physical and emotional exchange. This study describes shared attention bearing in mind levels of commitment and emotional tone, and it analyzes associated relationships with maternal sensitivity and the intensity of emotional expression in one-year-old children. The sample includes 12 mother-child dyads with the following inclusion criteria: only children, of ages between 12 and 14 months, living with both parents and attending a nursery. The instruments used were the Shared Attention Assessment, the Children's Emotional Expressions Assessment, and the Adult Sensitivity Scale (ASS). Results show episodes of shared attention between mother-child dyads at one year of age. A significant relationship between shared attention, levels of commitment, and maternal sensitivity was also found.

Key words: shared attention, emotional gesture expression, mother-child interactions, maternal sensitivity.
\end{abstract}

* Calle 15B sur \#53B-71, teléfono tel. 574 2853187, Medellín, Colombia, elizabethmunozlondono@gmail.com, chfarkas@gmail.com Este artículo de investigación contó con el apoyo de Fondo Nacional de Desarrollo Científico y Tecnológico (FONDECYT, n. N 1110087 , 1141118 y 1160110$)$. 


\title{
Relaçáo entre atençáo compartilhada mãe-filbo, sensibilidade materna e expressấo emocional gestual infantil
}

\begin{abstract}
Resumo
A atenção compartilhada refere-se a episódios nos quais a criança e seu cuidador estão focados intencionalmente em algum objeto ou atividade, apresentando trocas físicas e emocionais. Neste estudo, a atenção compartilhada foi descrita considerando as dimensões de nível de compromisso e tom emocional, e foi analisada sua relação com a sensibilidade materna e com a intensidade da expressão emocional gestual de crianças de um ano de idade. A amostra estava composta por 12 duplas mãe-filho, cujos critérios de inclusão eram que as crianças tivessem entre 12 e 14 meses de idade, que fossem filhos únicos, que vivessem com ambos os pais e que frequentassem o berçário. Os instrumentos utilizados foram a Avaliação de Atenção Compartilhada, a Avaliação da Expressão Emocional Gestual da Criança e a Escala de Sensibilidade do Adulto. Os resultados evidenciam episódios de atenção compartilhada em duplas mãe-filho com um ano de idade, além de uma relação significativa entre atenção compartilhada com o nível de compromisso e a sensibilidade materna.

Palavras-chave: atenção compartilhada, expressão emocional gestual, interação mãe-criança, sensibilidade materna.
\end{abstract}

\section{INTRODUCCIÓN}

La interacción entre el niño y la madre es el escenario para el desarrollo de la atención compartida, que se refiere a aquellos episodios en los que el niño y un cuidador significativo (por lo general, la madre) están mutuamente enfocados en algún objeto o situación (Mundy \& Sigman, 2006; Tomasello \& Todd, 1983). Durante dicho episodio, el niño, o la madre, hace una propuesta de intercambio a su compañero en la interacción (especialmente, le mira a la cara y luego retorna a la actividad). Esto último es esencial cuando aparece en el niño, ya que pone en evidencia que es consciente de la interacción en la que está tomando parte. Así, la atención compartida implica que ambos participantes, durante una interacción física y afectiva, atienden intencionalmente a un objeto o situación (Adamson \& Bakeman, 1991; Trevarthen \& Hubley, 1978).

Estas interacciones van evolucionando de manera paulatina, ya que en los primeros meses de vida el niño centra su interés solo en la madre y forma un sistema diádico; pero cuando el niño crece, va incorporando en su interacción un interés por los objetos y el mundo exterior, con el que produce un cambio en la relación a un sistema tríadico; es decir, se comparte la atención entre el niño, la madre y el objeto o situación (Baron Cohen, 1994; De Groote, Roeyers \& Striano, 2007). Luego, de los seis a nueve meses tiene lugar un estadio intermedio, denominado interacción pasiva, en el que madre y niño comparten objetos, pero este no muestra conciencia de la independencia de la madre (Bakeman \& Adamson, 1984; Butterworth, 2004). Posteriormente, entre los nueve y doce meses (estadio prelingüístico), los niños coordinan su atención entre ellos, la persona y un objeto o situación, y allí se inicia el reconocimiento de intenciones (Baron Cohen, 1995). Finalmente, alrededor de los doce meses se consolida la atención compartida en el niño (Acosta, 1996; Adamson y McArthur, 1995; Baron Cohen, 1994; Trevarthen, 1998). Teniendo en cuenta este último hallazgo, los niños que participan en el presente estudio tienen 12 meses de edad.

Diversos estudios han señalado la importancia de la atención compartida en el desarrollo del niño. En primer lugar, dicha atención se asocia con la adquisición de habilidades cognitivas, como identificar que los demás tienen objetivos, intenciones y estados atencionales - es decir, una intersubjetividad secundaria-(Adamson \& McArthur, 1995; Martínez, 2010; Trevarthen, 1998); habilidades que le permiten al niño aumentar su participación en los episodios de atención compartida y construir sus propias representaciones del mundo (Stern, 1997). Además, la atención compartida es fundamental para el desarrollo de la teoría de la mente, en la que el niño comprende la conducta de un otro, atribuyéndole intencionalidad y estados internos-como pensamientos y emociones-(Baron Cohen, 1994; 1995); y promueve el desarrollo de la comunicación gestual (Aravena et al., 2008; Galeote, Checa, Serrano \& Rey, 2004; Mundy et al., 2007; Tomasello \& Farrar, 1986).

Los resultados de escasas investigaciones realizadas en la población chilena evidencian la relevancia de la atención compartida en el niño, ya que han demostrado una correlación positiva entre el desarrollo comunicativo de los niños y 
la atención compartida (Aravena et al., 2008), así como la importancia del mecanismo materno de introducción y reorientación de la atención del niño para mantener y coordinar la atención compartida en la díada (Mendive, Bornstein \& Sebastian, 2013). Al respecto, la escasa evidencia de investigaciones chilenas motiva la necesidad de estudiar la atención compartida en dicha población, con la finalidad de aportar al avance a nivel teórico en el tema y caracterizar la formación de los episodios de atención compartida entre madre e hijo en el contexto chileno.

Por otra parte, la evaluación de la atención compartida suele hacerse en contextos de juego libre, ya que la mayoría de los estudios coinciden en que el juego es el mejor escenario para observar, evaluar y analizar la atención compartida entre madre e hijo, al ser una situación en donde se dan rutinas de interacción similares a la cotidianeidad (Mas, 2003). Sin embargo, su operacionalización difiere con respecto al foco de estudio y la conceptualización del constructo, ya que algunas codificaciones se centran exclusivamente en las habilidades del niño, otras solo en las de la madre, pocas en el proceso de la díada (Sadurní \& Pérez, 2008), y muy pocas en la relación entre el niño, la madre y el objeto (Bakeman \& Adamson, 1984; Camaioni, Aureli, Bellagamba \& Fogel, 2003).

También, se ha estudiado la manipulación de los objetos y los tipos de juego que desarrolla el niño con su madre (Dixon \& Smith, 2003; Zelazo \& Kearley, 1980) y la capacidad de representación del niño y la madre, es decir, que se focalizan en la interacción primaria y secundaria (Mas \& Añaños, 2010; Sadurní \& Pérez, 2008), donde se estudian los ritmos durante la interacción al observar la iniciativa y la finalización de los episodios de atención compartida (Aravena et al., 2008; Mendive, 2009; Mundy et al., 2007).

$\mathrm{Al}$ respecto, la presente investigación se enfoca en el tipo de interacción que ocurre entre la madre y el niño, considerándose dos dimensiones de la misma: (a) el nivel de compromiso de la díada, que se define como los momentos en donde los participantes comparten entre ellos y con el objeto u actividad, y al mismo tiempo interactúan intencionalmente; y (b) el tono emocional, que alude a la expresión emocional de cada participante a partir de intercambios afectivos observados en el lenguaje corporal, la expresión facial y el tono de la voz (Vallotton, 2004).

Asimismo, la sensibilidad materna y la expresión emocional gestual del niño son variables relevantes para la atención compartida debido a que, en dichos episodios, es importante contemplar los ritmos, las iniciativas y las repuestas -o falta de estas- que se presenten en la díada. Así, la sensibilidad materna implica que la madre perciba y responda adecuadamente a las señales del niño durante la interacción; mientras que las señales transmitidas por el niño durante los episodios de atención compartida se presentan principalmente a través de gestos -debido a su corta edad- que le sirven para expresar sus estados emocionales con respecto a los objetos o situaciones en que interactúa con la madre.

Específicamente, según Crittenden (2005), la sensibilidad materna es entendida como un constructo diádico que corresponde a cualquier patrón de conducta desplegado por el adulto con la intención de tranquilizar al infante, incrementar su comodidad y atender a sus señales; por lo que un adulto sensible sería aquel que toma conciencia, interpreta y responde de forma apropiada y contingente a las señales del niño (Ainsworth, Blehar, Waters \& Wall, 1978). Al respecto, es importante señalar que la sensibilidad materna ha sido relacionada con el desarrollo de la atención compartida (Ainsworth et al., 1978; Bigelow et al., 2009; DeWolff \& van IJzendoorn, 1997; Meins, 1997; Van den Boom, 1994), ya que las madres con una sensibilidad adecuada adaptan su comportamiento interactivo y foco de atención a las necesidades del niño, lo que permite que este pueda focalizarse en la interacción durante los episodios de atención compartida (Fein \& Fryer, 1995; Fiese, 1990), además de que promueve un mayor número de episodios de este tipo de atención en el niño, lo que favorece el aprendizaje, el lenguaje y la simbolización en sus primeros años de vida (Bigelow, MacLean \& Proctor, 2004; Kochanska \& Askan, 2004; Marfo, 1990).

Mientras que la expresión emocional gestual del niño es entendida como la capacidad que tiene de expresar estados internos (necesidades, estados de ánimo, motivaciones, emociones, etc.) de manera facial o corporal, además de su intención de interactuar con un otro (Gosselin, Kirouac \& Doré, 1995; Tronick, 1989; Zajonc 1989). Al respecto, cabe señalar que desde temprana edad, y durante el primer año de vida, la comunicación entre el niño y su madre se manifiesta en formas no verbales - como gestos o señales-(Lebovici, 1983), lo que quiere decir que los niños usan el lenguaje corporal y las expresiones gestuales para manifestar sus necesidades, sensaciones y sentimientos a sus cuidadores (Farkas, 2007). Esto demuestra que la madre le otorga al niño el papel de interlocutor y no solamente el de receptor (Acosta, 1996).

Adicionalmente, la expresión gestual de emociones juega un rol importante de regulación en las interacciones sociales, ya que le permite a las personas involucradas evaluar los estados emocionales del otro y ajustar su comportamiento a lo esperado (Gosselin, et al., 1995; Petit, 2009), además de generar ciertas respuestas en el interlocutor (Hendler, Kielmanowicz, Reingold \& Rotman, 2012). Sin embargo, la expresión de emociones en los niños presenta diferencias individuales -como en la expresión de la irritabilidad, 
la capacidad de consolabilidad, la actividad motora, la reactividad a los estímulos o la claridad de las señales para dar a conocer sus necesidades (Brazelton \& Cramer, 1993; Hendler et al., 2012)-, características que influyen en la intensidad de la expresión de las emociones y que afectan el modo en que ellos interactúan con sus madres (Lebovici, 1983).

Teniendo en cuenta lo expuesto, para el presente estudio surgieron dos objetivos principales: (a) describir la atención compartida de díadas madre-hijo cuando los niños tienen un año de edad; y (b) analizar la relación entre la atención compartida de las díadas madre-hijo y las variables sensibilidad materna e intensidad de la expresión emocional gestual del niño.

\section{MÉTODO}

\section{Diseño}

Se realizó un diseño exploratorio, transversal-ya que se realiza una sola medición en el tiempo-, descriptivo -porque describe la atención compartida de la díada en los niveles de compromiso y tono emocional- y correlacional -al profundizar sobre las relaciones entre la atención compartida, la sensibilidad de la madre y la intensidad de la expresión emocional gestual del niño-, con metodología cuantitativa.

\section{Participantes}

La muestra estuvo compuesta por 12 díadas madre-hijo seleccionadas a través de un muestreo no probabilístico intencional de un estudio longitudinal mayor. Los criterios de selección fueron que los niños (a) tuvieran entre 12 y 14 meses de edad, (b) fueran hijos únicos, (c) vivieran con ambos padres, (d) asistieran a sala cuna en Santiago de Chile, y (e) fueran ingresados a sala cuna después de los seis meses cumplidos. Estos criterios de selección buscaron favorecer la homogeneidad de la muestra e intentaron controlar la presencia de otros factores que interfirieran sobre las variables en estudio.

Así, las 12 díadas madre-hijo estuvieron compuestas por niños entre los 12 y 14 meses de edad $(M=12.42$, $D E=.669)$, de los cuales el $50 \%$ eran niños y el $50 \%$, niñas; y madres con una media de edad de 28.08 años $(D E$ $=5.728$, rango entre 19 y 35 años), distribuidas en tres categorías de sensibilidad (50\% en sensibilidad alta, 33.3\% en sensibilidad adecuada y $16.7 \%$ en sensibilidad baja), y un nivel socioeconómico (NSE) homogéneo, evaluado con el índice ESOMAR, en el cual se contempla el nivel educacional y ocupacional y donde el $33.3 \%$ corresponde a cada nivel socioeconómico de la díada (NSE bajo, medio y alto).

\section{Instrumentos}

Evaluación de la atención compartida. Para evaluar esta variable se hizo uso de una situación de juego libre entre la díada madre-niño, que fue observada y grabada durante un periodo de 5 minutos. La codificación de la atención compartida fue dividida en dos dimensiones, de acuerdo con lo propuesto por Vallotton (2004).

La primera dimensión se refiere al "nivel de compromiso", que comprende 5 niveles: compromiso compartido total (codificado como 4), que se presenta cuando ambos integrantes están comprometidos de manera intencional en la tarea o actividad y en el otro miembro de la díada; compromiso compartido parcial (codificado como 3 ), donde ambos miembros de la díada están comprometidos en la actividad que está teniendo lugar, pero, al mismo tiempo, no están interactuando en una misma tarea; compromiso pasivo (codificado como 2), donde un miembro de la díada observa al otro; no compromiso parcial (codificado como 1), donde ambos miembros de la díada atienden a sus propias actividades; y no compromiso (codificado como 0 ), donde uno o ambos miembros de la díada están completamente retirados en sí mismos, o distraídos por algo fuera de la situación de la actividad del juego libre.

La segunda dimensión, denominada "tono emocional", es evaluada principalmente mediante el uso de la expresión facial, el tono de voz y el lenguaje corporal. Para esta clasificación se tomaron como referencia los tonos emocionales básicos que propone Vallotton (2004): (a) tono emocional positivo, cuando un miembro de la díada está sonriendo, su tono de voz es amable, y su lenguaje corporal puede incluir aplausos o animaciones hacia el otro; (b) tono emocional neutro, que indica un tono de voz monótono y una expresión facial "plana", es decir, que el sujeto no sonríe, pero tampoco se irrita ni frunce el ceño; y (c) tono emocional negativo, cuando un miembro de la díada tiene un tono de voz frío y su expresión facial -con la boca, el ceño o sus cejas fruncidas- evidencia que se encuentra irritable, molesto, aburrido o asustado.

A partir de estos tres tonos emocionales se crearon seis niveles de tono emocional para la díada: (a) sincronía positiva (codificado como 5), cuando ambos miembros de la díada manifiestan un tono emocional positivo; (b) sincronía parcial positiva (codificado como 4), cuando un miembro de la díada manifiesta un tono emocional positivo, mientras el otro manifiesta un tono emocional neutro; (c) sincronía neutra (codificado como 3), cuando ambos miembros de la 
díada manifiestan un tono emocional neutro; (d) sincronía parcial negativa (codificado como 2), cuando un miembro de la díada manifiesta un tono emocional negativo, mientras el otro manifiesta un tono emocional neutro; (e) sincronía negativa (codificado como 1), cuando ambos miembros de la díada manifiestan un tono emocional negativo; y (f) asincronía (codificado como 0), cuando un miembro de la díada manifiesta un tono emocional positivo y el otro uno negativo.

Para la segmentación de los videos se utilizó el software ELAN. La codificación fue realizada en segmentos de tres segundos, debido a que distintas investigaciones señalan que la atención compartida de la díada se presenta cuando han pasado tres segundos continuos en la misma actividad (Tomasello \& Farrar, 1986; Vallotton, 2004). Para cada segmento se asignó un puntaje en nivel de compromiso ( $0-4$ puntos) y en tono emocional ( $0-5$ puntos), según los criterios previamente descritos.

Por último, se le calculó al codificador una confiabilidad inter-juez con la evaluación de un segundo codificador experto que revisaba el $30 \%$ de los segmentos codificados totales. A partir de esto, se obtuvo una confiabilidad de $95 \%$ para el nivel de compromiso, y de $100 \%$ para el tono emocional (niño, adulto y díada).

\section{Escala de sensibilidad del adulto (ESA, Santelices} et al., 2012). Esta evaluación consiste en una interacción filmada de juego libre entre el adulto y el niño de cinco minutos. Este instrumento cuenta con una rúbrica de evaluación compuesta por 19 ítems que evalúan las conductas sensibles de la madre hacia el niño. A partir del puntaje asignado a cada indicador, se obtienen tres escalas: (1) la escala de respuesta empática, que indica un adulto capaz de reconocer las señales del niño, además de interpretarlas y responder a sus demandas; (2) la escala de interacción lúdica, donde el adulto es capaz de relacionarse con el niño tomando en cuenta sus iniciativas e incorporándolas en el juego; y (3) la escala de expresión emocional, que alude a un adulto que manifiesta una actitud sensible y cálida frente a las necesidades y emociones del niño. Teniendo esto en cuenta, con el instrumento se ubica al adulto en las categorías de sensibilidad baja, adecuada o alta.

Esta prueba ha demostrado un adecuado acuerdo interjueces (Kappa de Cohen de .62), y una confiabilidad adecuada, con un coeficiente alfa de Cronbach de .93 en una muestra de 99 díadas de apoderados y personal educativo en interacción con niños de 10 y 33 meses (Santelices et al., 2012); así como una confiabilidad de .88 en una muestra de 104 madres en interacción con niños de 10-15 meses
(Farkas et al., 2015), y de .92 en la muestra de 12 díadas del presente estudio.

Evaluación de la expresión emocional gestual del niño (Farkas, Santelices \& Himmel, 2011). Este instrumento evalúa la gestualización del niño en situaciones que involucren la expresión de emociones. Específicamente, utiliza cuatro tareas apropiadas para la la edad: las dos primeras son gratificantes para el niño (tarea de éxito), y las dos siguientes implican una actividad frustrante (tarea de fracaso); esto con el fin de inducir en el niño emociones placenteras y displacenteras.

Toda esta situación es grabada y posteriormente codificada con el registro de los gestos implicados en la expresión de las emociones placenteras y displacenteras, de acuerdo con una pauta diseñada para tal fin; similar a la planteada por Ekman, Friesen y Hager (2002), Tracy y Matsumoto (2008), y Wallbott (1998). En este sentido, se selecciona la expresión más intensa de placer y displacer para cada niño, y cada una de ellas es codificada de acuerdo con la presencia o ausencia de 54 gestos: (a) faciales, que incluyen la frente (ej.: FA1, arrugas horizontales), cejas (ej.: FB1, cejas elevadas), ojos (ej.: FC4, ambos ojos cerrados) y boca (ej.: FD4, sonrisa con boca cerrada); y (b) corporales, que incluyen el tronco (ej.: CA1, pecho expandido), la posición y movimiento de la cabeza (ej.: CB1, cabeza inclinada hacia el lado), la posición de los hombros (ej.: CD1, hombros arriba), la posición y movimiento de brazos (ej.: CE3, brazos levantados, o CG1, movimientos de apertura), y la posición y movimiento de las manos (ej.: CF1, manos en puño, o $\mathrm{CH} 1$, aplaudir).

Con base en lo codificado, se obtienen puntajes de intensidad para la expresión facial ( $0-5$ puntos), corporal (0-8 puntos) y total (la suma de los dos anteriores: 1-13 puntos), donde a mayor puntaje, mayor intensidad de la expresión. La confiabilidad inter-juez del instrumento se evaluó mediante correlaciones entre seis codificadores y un Master Code, con indicadores entre .87 a .96 para gestos faciales, y .95 a .98 para gestos corporales (Muzard, Kwon, Espinosa, Vallotton \& Farkas, 2017).

\section{Procedimiento}

Las videograbaciones utilizadas en este estudio corresponden a datos secundarios de un estudio longitudinal mayor, en el que se analizó la relación de las expresiones emocionales de los niños con competencias parentales como la sensibilidad y la mentalización de apoderados y personal educativo con niños de 12 y 30 meses de edad. En dicho estudio se estableció un contacto inicial con distintas salas cuna de la ciudad de Santiago, en donde se les solicitaba 
su autorización para participar. Luego, se invitó a las díadas que contaban con los criterios de inclusión, se les presentó los objetivos del estudio, así como sus derechos como participantes, y se les solicitó la firma de una carta de consentimiento. Posteriormente, las madres llenaron un cuestionario sociodemográfico, y se procedió a filmar cinco minutos de juego libre con su hijo. En detalle, para evaluar su expresión emocional gestual, los niños realizaron cuatro tareas en presencia de la madre.

Las díadas participantes de este estudio corresponden al $13 \%$ de la muestra original y fueron seleccionadas a través de un muestreo intencional. Todas las filmaciones se realizaron en la sala cuna a la cual asistían los niños.

\section{Análisis de datos}

Para el primer objetivo del estudio, descripción de la atención compartida de las díadas, se realizaron análisis de frecuencia de sus dos dimensiones: nivel de compromiso (5 categorías) y tono emocional (6 categorías). Para estos análisis, se consideraron los segmentos totales (1183 segmentos de un total de 1200, cada uno de tres segundos de duración-el $1.4 \%$ del total de los segmentos corresponde a segmentos perdidos por posturas o recuadros que no permitían su codificación-), así como la descripción de las díadas. Además, se analizó la duración de los episodios de compromiso compartido total, ya que es la categoría que da cuenta de la capacidad de la díada para sostener su atención de manera compartida y continua.

Para analizar el segundo objetivo, relación de la atención compartida de las díadas con la sensibilidad materna y la intensidad de la expresión gestual del niño, se calculó la mediana tanto para el nivel de compromiso como para el tono emocional, esto debido a que, al ser escalas ordinales, no se conoce la distancia entre los valores, y porque la distribución de las díadas en las categorías es asimétrica; es por esto que se sugiere el uso de medianas en vez de medias (Sullivan \& Artino, 2013). Hecho esto, se pudo apreciar que todas las díadas obtenían la misma mediana en tono emocional, lo que impidió su análisis con otras variables; por tanto, para los análisis posteriores solo se tomó en cuenta el nivel de compromiso.

Después de esto, se realizó un análisis correlacional (Tau-b de Kendall) para analizar exploratoriamente la relación entre el nivel de compromiso, las variables de sensibilidad materna (puntaje total y de las tres escalas) y la intensidad de la expresión emocional gestual del niño (puntaje total para las expresiones emocionales de placer y displacer). Y, por último, se realizó un análisis de regresión jerárquica para estudiar el porcentaje de varianza explicada del nivel de compromiso de la díada, considerando la sensibilidad materna y la expresión emocional infantil, y controlando el NSE de la díada, ya que es una variable que influye en la sensibilidad materna (Farkas et al., 2015; Santelices et al., 2015) y en la expresión gestual emocional infantil (Muzard et al., 2017).

\section{RESULTADOS}

A continuación, se presentan los análisis descriptivos de la atención compartida de la díada, considerando el nivel de compromiso y el tono emocional. Posteriormente, se presentan los análisis correlacionales realizados entre el nivel de compromiso de la díada, la sensibilidad materna y la expresión emocional gestual de los niños. Y, por último, se revisa el análisis de regresión realizado para determinar el porcentaje de varianza explicada por las variables de la madre y el niño para el nivel de compromiso de la díada.

\section{Análisis descriptivos de la atención compartida de la díada}

Con respecto a los cinco niveles evaluados en la dimensión "nivel de compromiso", los resultados mostraron que el compromiso pasivo fue el más frecuente $(42.1 \%$ de los segmentos), seguido por el nivel compromiso compartido parcial (25.8 \% de los segmentos). Por otra parte, si se considera que compromiso compartido total es el nivel que más se acerca a la definición de atención compartida, es de destacar que se encontró una frecuencia de tan solo 19.9 \% para esta dimensión en los segmentos analizados (véase Tabla 1).

Tabla 1

Distribución de frecuencia para el nivel de compromiso

\begin{tabular}{lcc}
\hline \multicolumn{1}{c}{ Nivel compromiso } & Frecuencia & Porcentaje \\
\hline No compromiso & 25 & 2.1 \\
No compromiso parcial & 119 & 10.1 \\
Compromiso pasivo & 498 & 42.1 \\
Compromiso compartido parcial & 305 & 25.8 \\
Compromiso compartido total & 236 & 19.9 \\
Total & 1183 & 100.0
\end{tabular}

Nota. Se presenta el análisis con base en el total de segmentos analizados en las 12 díadas.

Con respecto al nivel de compromiso compartido total, se analizó el tiempo de duración de estos episodios, teniendo en cuenta la capacidad de la díada para sostener su atención de forma continua. Este tiempo osciló entre 3 y 75 segundos (1 a 25 segmentos continuados). Además, se observó que la mayor frecuencia $(18.4 \%)$ corresponde a los episodios de seis segundos de duración (véase Figura 1). 


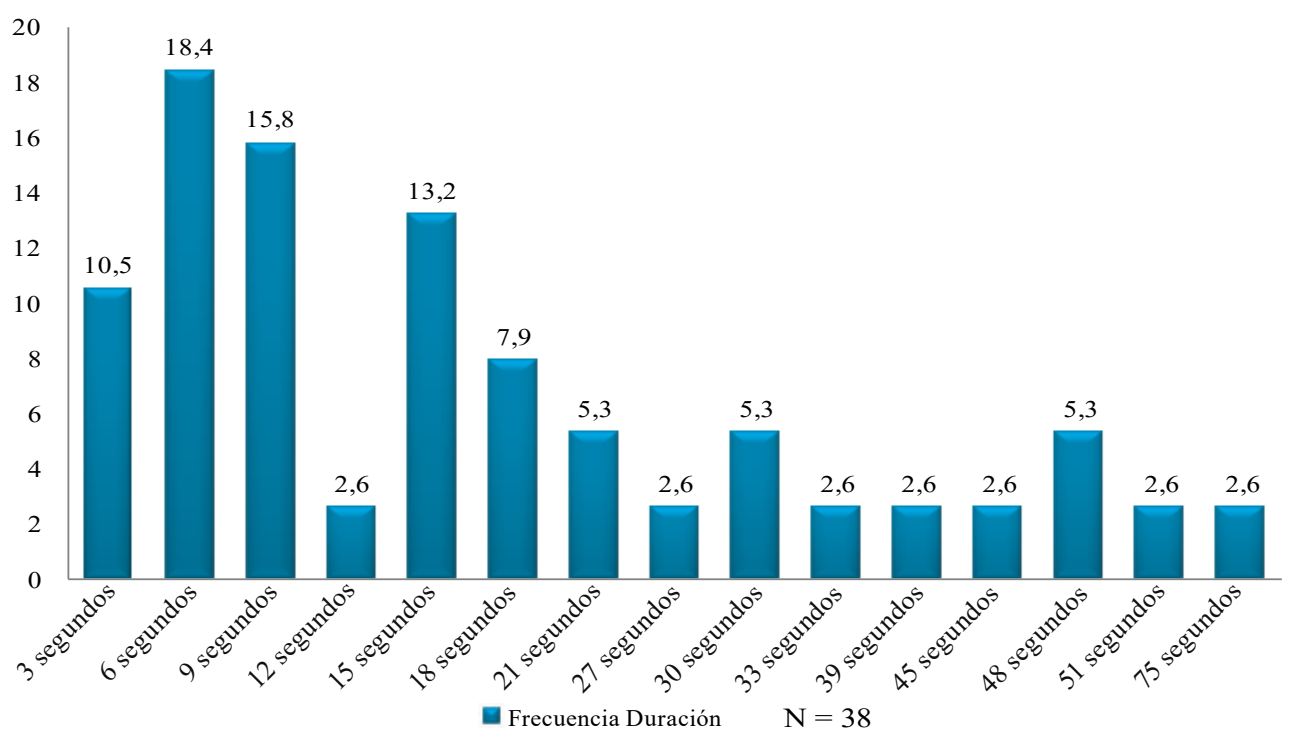

Figura 1. Frecuencia tiempo de duración para el nivel de compromiso compartido total.

Tabla 2

Distribución de frecuencia para el tono emocional del niño y la madre

\begin{tabular}{lcccc}
\hline \multirow{2}{*}{ Tono emocional } & \multicolumn{2}{c}{ Niño } & \multicolumn{2}{c}{ Madre } \\
\cline { 2 - 5 } & Frecuencia & Porcentaje & Precuencia & .2 \\
\hline Negativo & 13 & 1.2 & 2 & 75.8 \\
Neutro & 944 & 84.1 & 903 & 24.0 \\
Positivo & 166 & 14.8 & 286 & 100.0 \\
Total & 1123 & 100.0 & 1191 &
\end{tabular}

Nota. Se presenta el análisis con base en el total de segmentos analizados en las 12 díadas.

Por otra parte, con respecto al "tono emocional", se observó que tanto en el niño como en la madre predominó un tono emocional neutro $(84.1 \%$ en el niño y $75.8 \%$ en la madre), seguido del tono emocional positivo ( $14.8 \%$ en el niño y $24 \%$ en la madre). Además, el tono emocional positivo fue relativamente más frecuente en la madre que en el niño (véase Tabla 2). Y, por último, los resultados mostraron que el nivel de tono emocional más predominante en la díada fue el de sincronía neutra (70.9\% de los segmentos), seguido por el de sincronía parcial positiva (17.9\%) (véase Tabla 3).

Con respecto a las 12 díadas evaluadas, en nueve de ellas se evidenció una tendencia predominante del nivel de compromiso pasivo, seguido de los niveles de compromiso compartido parcial y compartido total (véase Figura 2). Esto indica que existe un patrón común según la frecuencia de los tres niveles de compromiso más predominantes durante la interacción. Además, en cuanto al tono emocional, se evidenció en nueve díadas una predominancia de la frecuencia de la sincronía neutra, seguida de la sincronía parcial positiva y la sincronía positiva (véase Figura 3). Finalmente, siete díadas presentaron un patrón común tanto para el nivel de compromiso, con presencia de compromiso pasivo, seguido de compromiso compartido parcial y compartido total; como para el tono emocional, con presencia de sincronía neutra, seguida de la sincronía parcial positiva y la sincronía positiva (atención compartida).

Tabla 3

Distribución de frecuencia para el tono emocional de la díada

\begin{tabular}{lcc}
\hline \multicolumn{1}{c}{ Tono emocional } & Frecuencia & Porcentaje \\
\hline Asincronía & 1 & 0.1 \\
Sincronía negativa & 1 & 0.1 \\
Sincronía parcial negativa & 14 & 1.3 \\
Sincronía neutra & 794 & 70.9 \\
Sincronía parcial positiva & 201 & 17.9 \\
Sincronía positiva & 109 & 9.7 \\
Total & 1120 & 100.0 \\
\hline
\end{tabular}

Nota. Para el tono emocional de la díada se consideran en total 1120 segmentos. 


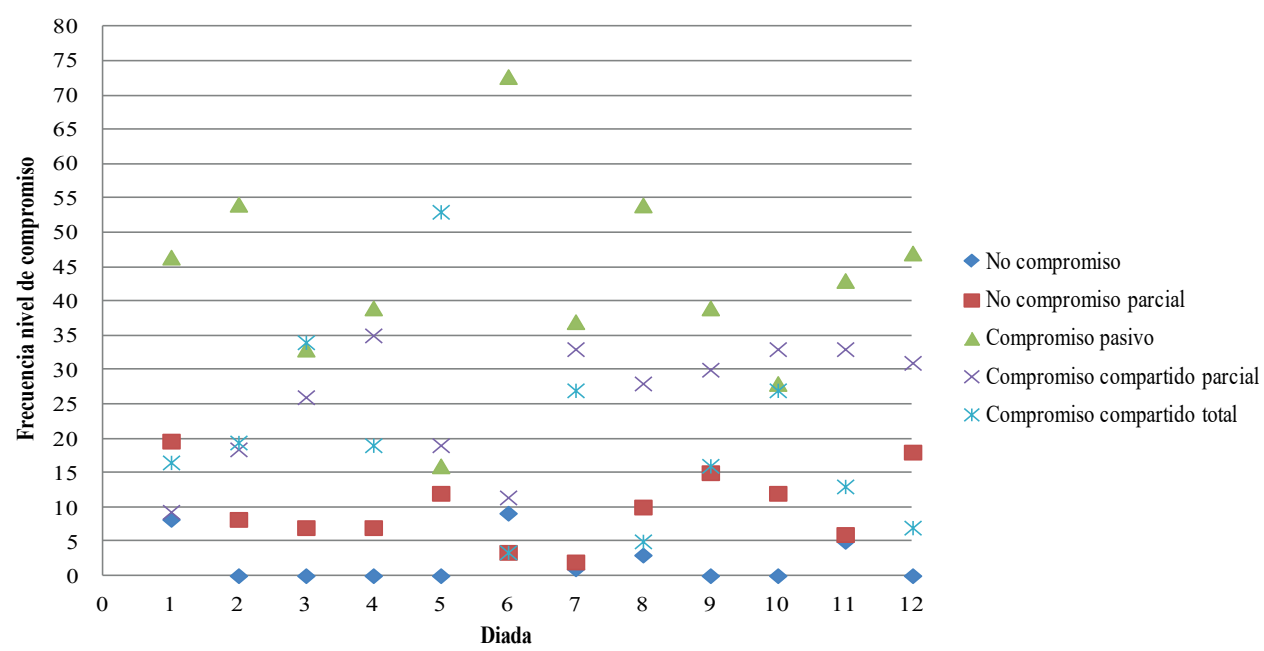

Figura 2. Dispersión de frecuencias para el nivel de compromiso de la díada.

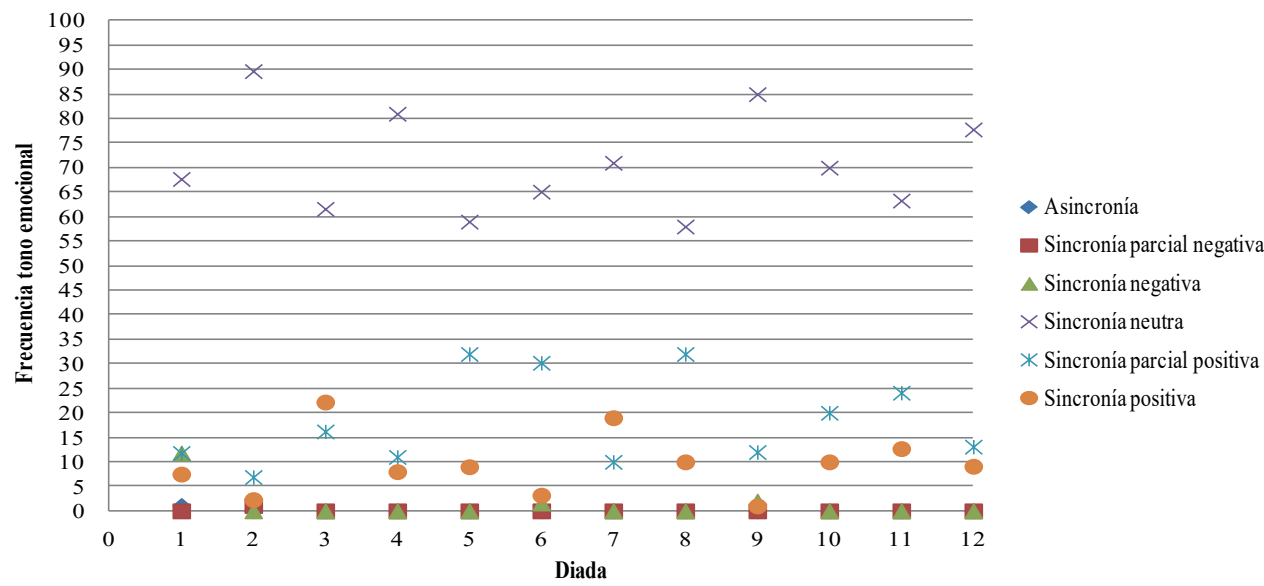

Figura 3. Dispersión de frecuencias para el tono emocional de la díada.

Análisis correlacionales entre el nivel de compromiso de la díada, la sensibilidad materna y la expresión emocional gestual

Se encontró una correlación significativa entre la sensibilidad materna total y el nivel de compromiso de la díada $\left(r_{b}=.611, p=.014\right)$ que indica que, a mayor sensibilidad materna, mayor nivel de compromiso en la díada; y, específicamente, un predominio de los niveles de compromiso compartido parcial y total. En cuanto a las escalas, un mayor puntaje en interacción lúdica correlacionó significativamente con un nivel más alto de compromiso $\left(r_{b}=.688, p=.008\right)$, al igual que con un mayor puntaje en expresión emocional $\left(r_{b}=.508, p=.046\right)$. Y, por último, no se evidenciaron correlaciones significativas entre el nivel de compromiso de la díada y las intensidades de expresión gestual de placer y displacer (véase Tabla 4).
Análisis de regresión para el nivel de compromiso de la díada.

Para analizar el porcentaje de varianza explicada por las variables de la madre y el niño consideradas en este estudio, se llevó a cabo un análisis de regresión jerárquica para el nivel de compromiso. En primer lugar, se ingresó el NSE de la díada como variable de control, luego el puntaje en sensibilidad total (modelo 1) y, finalmente, la intensidad para expresar placer y frustración en el niño (modelo 2).

Para el nivel de compromiso de la díada, la sensibilidad materna resultó ser el único predictor significativo $(\beta=$ $.932, t=3.051, p=.014)$, dando cuenta de un $49.6 \%$ de la varianza (véase Tabla 5, modelo 1 ), una vez controlado el NSE. Las intensidades de expresión de placer y frustración del niño no fueron un predictor significativo; y el modelo 1 resulto ser el único significativo $(F=4.888, p=.037)$. 
Tabla 4

Correlaciones entre el nivel de compromiso de la diada, la sensibilidad materna y la expresión gestual infantil

\begin{tabular}{lcc}
\hline & \multicolumn{2}{c}{ Nivel de compromiso de la díada } \\
\cline { 2 - 3 } \multicolumn{1}{c}{ Sensibilidad materna } & Coeficiente de correlación (rb) & Sig. \\
\hline Escala de respuesta empática & & \\
Escala de interacción lúdica & .363 & .152 \\
Escala de expresión emocional & $.688^{* *}$ & .008 \\
Puntaje total sensibilidad materna & $.508^{*}$ & .046 \\
$\quad$ Expresión gestual infantil & $.611^{*}$ & .014 \\
Intensidad total expresión de placer & & .115 \\
Intensidad total expresión de frustración & .422 & .871 \\
\hline
\end{tabular}

Nota. $* * \mathrm{p} \leq .01, * \mathrm{p} \leq .05$

Tabla 5

Regresión jerárquica para el nivel de compromiso de la díada, considerando la sensibilidad materna y la expresión emocional del niño

\begin{tabular}{|c|c|c|c|c|c|c|}
\hline & \multicolumn{3}{|c|}{ Modelo 1} & \multicolumn{3}{|c|}{ Modelo 2} \\
\hline & $\mathrm{B}$ & SE B & $\beta$ & $\mathrm{B}$ & SE B & $\beta$ \\
\hline Constante & .056 & .820 & & -1.891 & 1.274 & \\
\hline NSE & -.358 & .242 & -.453 & -.541 & .251 & $-.684 \sim$ \\
\hline Sensibilidad total & 1.381 & .453 & $.932 *$ & 2.244 & .700 & $1.515^{*}$ \\
\hline Intensidad expresión placer niño & & & & -.319 & .215 & -.566 \\
\hline Intensidad expresión frustración niño & & & & .213 & .125 & .400 \\
\hline $\mathrm{R}^{2}$ & & .521 & & & .684 & \\
\hline Cambio en $\mathrm{R}^{2}$ & & $.496^{*}$ & & & .163 & \\
\hline $\mathrm{F}$ & & $4.888 *$ & & & $3.788 \sim$ & \\
\hline
\end{tabular}

Nota. $* \mathrm{p}<.05, \sim \mathrm{p}<.09$.

\section{DISCUSIÓN}

Considerando la importancia de estudiar las situaciones que se presentan en la interacción entre madre e hijo durante el primer año de vida, y teniendo en cuenta algunas características de la díada que influyen en las interacciones, el presente trabajo brinda un aporte significativo al estado de la investigación al estudiar la relación de la atención compartida de la díada con la sensibilidad materna y la expresión emocional gestual infantil -variables que teóricamente están relacionadas, pero que no han sido relacionadas en investigaciones empíricas-- Inicialmente, los resultados de la investigación evidenciaron la factibilidad de estudiar la atención compartida a través de dos dimensiones: el nivel de compromiso y el tono emocional; dimensiones que permitieron operacionalizar y dar cuenta con mayor claridad y precisión del concepto de atención compartida.

Específicamente, los resultados descriptivos del estudio mostraron que el nivel de compromiso pasivo y el tono emocional de sincronía neutra fueron los más frecuentes en las díadas durante su interacción en el juego libre; mientras que los niveles correspondientes a la atención compartida de calidad, es decir, el compromiso compartido total y la sincronía positiva en el tono emocional, fueron menos frecuentes, a pesar de que sí estuvieron presentes. Esto concuerda con las investigaciones que sugieren que a los 12 meses ya existen las habilidades cognitivas necesarias para la atención compartida en el niño (Butterworth, 2004; Trevarthen, 1998).

Por otra parte, en las observaciones realizadas se pudo apreciar que los niños se dedicaban principalmente a tener relaciones con los objetos, más que con sus madres, por lo que se presentaba un sistema diádico (sujeto-objeto o sujeto-situación); mientras que la madre era el participante que observaba las interacciones del niño y propiciaba episodios de atención compartida. Resultado coherente con estudios en los que se ha señalado que en las interacciones lúdicas se requiere de la presencia de un otro que propicie y facilite el espacio para que el niño desarrolle y autorregule sus interacciones (Winnicott, 1971). 
A la vez, el hecho que un adulto esté constantemente introduciendo objetos en el juego del niño durante la interacción tiende a impedir el desarrollo de la atención compartida (Gamer \& Landry, 1994; Mas \& Añaños, 2010). Por otra parte, los episodios de atención compartida no se presentaron durante todo el tiempo de la interacción; aspecto esperable, debido a que al año de edad la atención del niño oscila entre explorar el ambiente y tener episodios de atención compartida con la madre, y que es solo posteriormente y de manera gradual que se desarrollan las capacidades para que el niño participe de un mayor número de situaciones de atención compartida (Richards \& Casey, 1992; Richards \& Turner, 2001).

Por otra parte, también se encontró que la duración de los episodios de nivel de compromiso compartido total evidenció una baja duración de los mismos, lo que se relaciona con la etapa de desarrollo en la cual se encuentra el niño, ya que mientras más pequeño es el niño, más cortos son los episodios interactivos observados; y que a medida que el niño crezca, aumentará la duración de los episodios de la atención compartida y, en consecuencia, potenciará otras habilidades -como la concentración y la regulación-que involucran al niño en una atención sostenida (Richards \& Casey, 1992; Richards \& Turner, 2001).

Asimismo, los resultados que se evidenciaron en la frecuencia de cada uno de los niveles presentes de la atención compartida son un aporte importante de la investigación, en tanto que mostraron un patrón común de distribución en nueve de las díadas analizadas. Así, específicamente, se presentó una predominancia del nivel compromiso pasivo en el nivel de compromiso, seguido del compromiso compartido parcial y el compromiso compartido total; mientras que para el tono emocional hubo una predominancia de la sincronía neutra, seguida de la sincronía parcial positiva y la sincronía positiva. Este resultado muestra que la presencia de un compromiso pasivo va generalmente acompañada de una sincronía neutra, y cuando se presenta compromiso compartido parcial o total, estos van acompañados de una sincronía parcial positiva o positiva. Esto indica que hay un conjunto de ritmos dentro de cada secuencia de juego que es rápidamente establecido y reconocido por cada participante en torno a los tiempos, la duración, la forma de compartir y los tonos emocionales presentes. Esto mismo permitirá aprendizajes a cada uno de los participantes a partir de las interacciones reciprocas (Stern, 1997).

Ahora bien, con respecto a la pregunta de la relación entre la atención compartida de la díada, la sensibilidad materna y la expresión emocional gestual infantil, los resultados reportaron una relación significativa entre la atención compartida -específicamente, el nivel de compromiso-, y la sensibilidad de la madre; y que no hubo tal significancia con la intensidad de la expresión emocional gestual del niño. Esto quiere decir que las madres que presentaron una sensibilidad mayor estaban más atentas a los intercambios que se presentaban con el niño y los objetos o situaciones, lo que contribuyó a que los episodios de atención compartida se presentaran con mayor frecuencia. Aspecto que se ha evidenciado en investigaciones previas (Bigelow et al., 2009; Coppola, Vaughn, Cassiba \& Costantini, 2006; Fein $\&$ Fryer, 1995; Fiese, 1990). Adicionalmente, cabe resaltar que la sensibilidad es una competencia materna relevante, y que a través de su relación con la atención compartida de la díada contribuye al desarrollo del niño a nivel biológico, psíquico y emocional (Ainsworth, et al., 1978; Meins, 1997; Stern, 1997).

Cabe mencionar que la falta de resultados con respecto a la expresión emocional gestual del niño puede deberse a la corta edad de los participantes, puesto que la relación que se pretendía encontrar puede ser observada en edades más avanzadas; es esto, o que posiblemente dicha variable guarde relación más bien con la dimensión de tono emocional de la díada, aspecto que no pudo ser incorporado en los análisis de este estudio. Futuros estudios con muestras de mayor tamaño y niños de distintas edades darán mayor claridad a esta pregunta.

Finalmente, en este estudio existen algunos limitantes, como el pequeño tamaño muestral y la poca variabilidad resultante en la atención compartida de las díadas, lo que no permitió incluir la dimensión de tono emocional en los análisis correlacionales, y por lo que los resultados deben ser tomados con cautela. Para evitar esto, futuros estudios en esta línea deben considerar muestras de mayor tamaño, con niños de distintas edades, y contemplar otras variables que puedan influir en la atención compartida, como el lenguaje empleado durante las interacciones, las características de personalidad y salud mental de la madre, entre otras.

No obstante las limitaciones mencionadas, los resultados obtenidos resultan interesantes, ya que evidencian cómo es que se manifiesta la atención compartida en díadas chilenas, así como la factibilidad de estudiarla a través de su operacionalización en las dimensiones de nivel de compromiso y tono emocional. Adicionalmente, su relación con la sensibilidad materna aporta una competencia que puede ser trabajada en intervenciones clínicas desde temprana edad del niño, y esto influirá positivamente en el desarrollo de la atención compartida madre-hijo, variable de gran relevancia para el futuro desarrollo cognitivo y emocional del infante. 


\section{REFERENCIAS}

Acosta, V. (1996). La evaluación del lenguaje: Teoría y práctica del proceso de evaluación en la conducta infantil (pp. 3351). Málaga: Aljibe.

Adamson, L., \& Bakeman, R. (1991). The development of shared attention during infancy. Annals of Child Development, 8, 1-41.

Adamson, L., \& McArthur, D. (1995). Joint Attention, affect, and culture. En C. Moore \& P. Dunham (Eds.), Joint attention: Its origins and role in development (pp. 205-221). New Jersey: Lawrence Erlbaum Associates Publishers.

Ainsworth, M., Blehar, M., Waters, E., \& Wall, S. (1978). Patterns of attachment: A psychological study of the strange situation. USA: Lawrence Erlbaum Associates.

Aravena, P., Loyola, R., Montero, C., Morales, K., Puebla, M., \& Maggiolo, M. (2008). Características de la atención conjunta y del desarrollo comunicativo en lactantes sanos entre 12 y 15 meses de edad (Tesis magister). Santiago, Chile: Universidad de Chile. Facultad de Medicina. Escuela de Fonoaudiología.

Bakeman, R., \& Adamson, L. (1984). Coordinating attention to people and objects in mother infant and peerinfant interaction. Child Development, 55, 1278-1289. doi:10.2307/1129997

Baron Cohen, S. (1994). How to build a baby that can read minds: Cognitive mechanisms in mindreading. Current Psychology of Cognition, 13, 513-552.

Baron Cohen, S. (1995). Mindblindness: An essay on autism and theory of mind. Boston: MIT Press/Bradford Books.

Bigelow, A. E., MacLean, K., \& Proctor, J. (2004). The role of joint attention in the development of infants' play with objects. Developmental Science, 7, 518-526. doi:10.1111/ j.1467-7687.2004.00375.x

Bigelow, A. E., MacLean, K., Proctor, J., Myatt, T., Gillis, R., \& Power, M. (2009). Maternal sensitivity throughout infancy: Continuity and relation to attachment security. Infant Behavior and Development, 2, 50-60. doi:10.1016/j. infbeh.2009.10.009

Brazelton, B., \& Cramer, B. (1993). La relación más temprana. Padres, bebé y el drama del apego inicial. ( $1^{\mathrm{a}}$ ed.). Barcelona: Paidós.

Butterworth, G. (2004). Joint visual attention in Infancy. En G. Bremner, \& A. Fogerl (Eds.), Blackwell Handbook of Infant Development (pp. 213-240). Australia: Blackwell Publishing. Doi: 10.1002/9780470996348.ch8

Camaioni, L., Aureli, T., Bellagamba, F., \& Fogel, A. (2003). A longitudinal examination of the transition to symbolic communication in the second year of life. Infant and Child Development, 12, 1-26. doi:10.1002/icd.333.

Coppola, G., Vaughn, B. E., Cassibba, R., \& Costantini, A. (2006). The attachment script representation procedure in an Italian sample: Associations with adult attachment interview scales and with maternal sensitivity. Attachment \& Human Development, 8(3), 209-219. doi:10.1080/14616730600856065.

Crittenden, P. (2005). Care-Index Para Toddlers: Manual de Codificación. Family Relations Institute. Miami, EE. UU.

De Groote, I., Roeyers, H., \& Striano, T. (2007). Gaze following as a function of affective expression in 3-, 6- and 9-monthold infants. Infant Behavior and Development, 30, 492-498. Doi:10.1016/j.infbeh.2006.12.009

DeWolff, M., \& van IJzendoorn, M. H. (1997). Sensitivity and attachment: A meta-analysis on parental antecedents of infant attachment. Child Development, 68, 571-591.

Dixon, W. E, \& Smith, P. H. (2003). Who's controlling whom? Infant contribution to maternal play behaviour. Infant and Child Development, 12, 177-195. doi:10.1016/S01636383(98)91596-X

Ekman, P., Friesen, W. V., \& Hager, J. C. (2002). The facial action coding system (2nd ed.). Salt Lake City, UT: Research Nexus eBook.

Farkas, C. (2007). Comunicación gestual en la infancia temprana: Una revisión de su desarrollo, relación con el lenguaje e implicancias de su intervención. Psykhe, 16, 107-115. doi:10.4067/S0718-22282007000200009

Farkas, C., Carvacho, C., Galleguillos, F., Montoya, F., León, F., Santelices, M. P., \& Himmel, E. (2015). Estudio comparativo de la sensibilidad entre madres y personal educativo en interacción con niños y niñas de un año de edad. Perfiles Educativos, 37(148), 16-33.

Farkas, C., Santelices, M. P., \& Himmel, E. (2011). Análisis desde una perspectiva evolutiva y cultural del uso de la comunicación gestual en infantes y pre-escolares, en la expresión y comprensión de los estados internos y su impacto en el desarrollo socio-emocional de los niños. Proyecto Fondecyt $N^{\circ}$ 1110087. Santiago, Chile: Pontificia Universidad Católica de Chile, Escuela de Psicología.

Fein, G., \& Fryer, M. (1995). Maternal contributions to early symbolic play competence. Developmental Review, 15, 367-381. doi:10.1006/drev.1995.1014

Fiese, B. (1990). Playful relationships: A contextual analysis of mother-toddler interaction and symbolic play. Child Development, 61, 1648-1656. doi:10.1111/j.1467-8624.1990.tb02891.x

Galeote, M., Checa, E., Serrano, A., \& Rey, R. (2004). De la evaluación a la intervención: Atención conjunta, directiva y desarrollo del lenguaje en niños con síndrome de down. Sindrome de Down, 21, 114-121.

Gamer, P. W., \& Landry S. H. (1994). Effects of maternal attention-directing strategies on preterm infants' affective expressions during joint toy play. Infant Behavior and Development, 17, 15-22. 
Gosselin, P., Kirouac, G., \& Doré, F. Y. (1995). Components and recognition of facial expression in the communication of emotion by actors. Journal of Personality and Social Psychology, 68(1), 83-96. Doi: 10.1037/0022-3514.68.1.83

Hendler, L., Kielmanowicz, R., Reingold, M., \& Rotman, M. (2012). El método de observación de bebés y sus aportes al vínculo temprano. Infancia \& compañia: La vida emocional del bebé y del niño pequeño. (pp. 35-49). Buenos Aires: Lugar Editorial.

Kochanska, G., \& Askan, N. (2004). Development of mutual responsiveness between parents and their young children. Child Development, 75, 1657-1676. Doi: 10.1111/j.1467-8624.2004.00808.x

Lebovici, S. (1983). El lactante, su madre y el psicoanálisis. Las interacciones precoces. Buenos aires: Amorrortu Editores.

Marfo, K. (1990). Maternal directiveness in interactions with mentally handicapped children: An analytical commentary. Journal of Child Psychology and Psychiatry, 31, 531-549. doi:10.1111/j.1469-7610.1990.tb00796.x

Martínez, M. (2010). De la intersubjetividad primaria a la secundaria: Qué percibe el bebé cuando mira al adulto (Tesis magíster). Madrid, España: Universidad Autónoma de Madrid.

Mas, M. T. (2003). L'atenció conjunta dels 10 als 28 mesos d'edat de l'infant (Tesis doctoral). Barcelona, España: Universidad Autónoma de Barcelona, Departamento de Psicología Básica, Evolutiva y de Educación.

Mas, M. T., \& Añaños, E. (2010). Relación entre atención compartida y atención sostenida en situación de juego tríadico. En E. Añaños, S. Estaún \& Mas, M. T. (Eds.), La atención (VI): Un enfoque interdisciplinar (pp. 161-166). Barcelona: Montflorit Edicions.

Meins, E. (1997). Security of attachment and the social development of cognition. Hove, UK: Psychology Press.

Mendive, S. (2009). How do 9-month old infants attain joint engagement when interacting with their mothers. The role of maternal attention-directing strategies (Tesis doctoral). Santiago, Chile: Pontificia Universidad Católica de Chile, Escuela de Psicología.

Mendive, S., Bornstein, M. H., \& Sebastian, C. (2013). The role of maternal attention-directing strategies in 9-month-old infants attaining joint engagement. Infant Behavior Development, 36(1), 115-123. doi: 10.1016/j. infbeh.2012.10.002

Mundy, P., Block, J., Delgado, C., Van Hecke, A. V., Parlade, M. V., \& Pomares, Y. (2007). Individual differences and the development of joint attention in infancy. Child Development, 78(3), 938-954. doi:10.1111/j.14678624.2007.01042.x

Mundy, P., \& Sigman, M. (2006). Joint attention, social competence, and developmental psychopathology. En D. Cicchetti \& D.J. Cohen. (Eds), Developmental psychopathology 2. Vol. 1 (pp. 293-332). New jersey, John Wiley \& Sons, Inc.
Muzard, A.; Kwon, A.; Espinosa, N.; Vallotton, C., \& Farkas, C. (2017). Infants' emotional expression: A crosscultural study between Chile and U.S.A. Infant and Child Development, 26(6), 3-5. doi:10.1002/icd.2033

Petit, M. (2009). El arte de la lectura en tiempos de crisis. Mexico: Editorial Océano.

Richards, J. E., \& Casey, B. J. (1992). Development of sustained visual attention in the human infant. En A B. A. Campbell, H. Hayne \& R. Richardson (Eds.) Attention and information processing in infants and adults (pp. 30-60). Hillsdale: Erlbaum.

Richards, J. E., \& Turner, E. D. (2001). Extended visual fixation and distractibility in children from six to twentyfour months of age. Child Development, 72(4), 963-972. doi:10.1111/1467-8624.00328

Sadurní, M., \& Pérez, M. (2008). La observación de la capacidad representacional en la díada madre-niño: Una nueva propuesta metodológica. Revista Psicológica Herediana, 2(2), 115-132.

Santelices, M. P., Carvacho, C., Farkas, C., León, F., Galleguillos, F., \& Himmel, E. (2012). Medición de la sensibilidad del adulto con niños de 6 a 36 meses de edad: Construcción y análisis preliminar de la Escala de Sensibilidad del Adulto, E.S.A. Terapia Psicológica, 30, 19-29. doi:10.4067/S071848082012000300003

Santelices, M. P., Farkas, C., Montoya, M. F., Galleguillos, F., Carvacho, C., Fernández, A., Morales, L., Taboada, C. \& Himmel E. (2015). Factores predictivos de sensibilidad materna en infancia temprana. Psicoperspectivas, 14(1), 6676. doi:10.5027/PSICOPERSPECTIVAS-VOL14ISSUE1FULLTEXT-441

Stern, D. (1997). La constelación maternal. La psicoterapia en las relaciones entre padres e hijos. ( $1^{\mathrm{a}} \mathrm{ed}$.). Buenos Aires: Paidós.

Sullivan, G. M. \& Artino, A. R. (2013). Analyzing and interpreting data from likert-type scales. Journal of Graduate Medical Education, 5(4), 541-542. doi:10.4300/ JGME-5-4-18

Tomasello, M., \& Todd, J. (1983). Joint attention and lexical acquisition style. First Language, 4, 197-212. doi:10.1177/014272378300401202

Tomasello, M., \& Farrar, M. (1986). Joint attention and early language. Child Development, 57, 1454-1463. doi:10.1111/j.1467-8624.1986.tb00470.x

Tracy, J. L., \& Matsumoto, D. (2008). The spontaneous expression of pride and shame: Evidence for biologically innate nonverbal displays. PNAS, 105(33), 11655-11660.

Trevarthen, C. (1998). The concept and foundations of infant intersubjectivity. En S. Braten (Eds.), Intersubjective communication and emotion in early ontogeny. Cambridge: Cambridge University Press. 
Trevarthen, C., \& Hubley, P. (1978). Secondary intersubjectivity: Confidence, confiders, and acts of meaning in the first years of life. En A Lock (Eds.), Actions, gesture and symbol. New York: Academic Press.

Tronick, E. Z. (1989). Emotions and emotional communication in infants. American Psychologist, 44, 112-119. Doi: 10.1037/0003-066X.44.2.112

Vallotton, C. (2004). Effects of symbolic gestures as a caregiving tool: Children's social and language development and mothers' perceptions and behavior (Tesis doctoral). California: Universidad de California.

Van den Boom, D. (1994). The influence of temperament and mothering on attachment and exploration. Child Development, 65, 1457-1477. doi:10.2307/1131511
Wallbott, H. G. (1998). Bodily expression of emotion. European Journal of Social Psychology, 28, 879-896. Doi: 10.1002/(SICI)1099-0992(1998110)28:6\%3C879::AIDEJSP901\%3E3.0.CO;2-W

Winnicott, D. (1971). Los procesos de maduración y el ambiente facilitador. Estudio para una teoría del desarrollo emocional. Buenos Aires: Paidós.

Zajonc, R. B. (1989). Styles of explanation in social psychology. Journal of Social Psychology, 19, 345-368. Doi: 10.1002/ ejsp.2420190503

Zelazo, P., \& Kearsley, R. (1980). The emergence of functional play in infants: Evidence for a major cognitive transition. Journal of Applied Developmental Psychology, 1, 95-117. doi:10.1016/0193-3973(80)90002-7 